\title{
Pitch evaluation of gratings based on a digital image correlation technique
}

\author{
Yancong Lu, Wei Jia, Chunlong Wei, Junjie Yu, Shubin Li, Yanyang Li, Minkang Li, \\ Jucheng Qiu, Shaoqin Wang, Changhe Zhou* \\ University of Chinese Academy of Sciences, Shanghai Institute of Optics and Fine Mechanics, Laboratory of \\ Information Optics and Optoelectronics Techniques, Qinghe Road NO.390, Shanghai, China, 201800
}

\begin{abstract}
The digital image correlation (DIC) technique used for metrological grating evaluation is presented in this paper. A CCD camera is used to acquire the grating image, and the DIC technique together with the peak-position detection method is used to evaluate the grating pitches. The theoretical analysis and simulations are performed to confirm that the performance of our technique is as accurate as the Fourier transform (FT) technique, and is capable of noise resistance. As an example, the uniformity of the grating fabricated in our laboratory is measured using this method. The experimental results show that this grating has a peak-to-valley uniformity of $48 \mathrm{~nm}$ during a long range of $35 \mathrm{~mm}$, and our technique has a higher repeatability than the FT technique in our measurement strategy. This work should be of great significance for the evaluation of metrological grating for optical encoders.
\end{abstract}

Keywords: pitch evaluation; grating; digital image correlation; uniformity.

*Changhe Zhou, E-mail: chazhou@mail.shcnc.ac.cn

\section{Introduction}

Optical diffraction (OD) technique and scanning probe microscopy (SPM) have been successfully applied to measure one-dimensional diffraction grating pitches in the international comparison (WGDM-7 DG) [1-5]. However, these techniques are not suitable for the measurement of metrological grating pitches because of the long length of metrological gratings. The OD technique is able to provide the average value of the grating pitches, but it cannot provide the grating pitch uniformity that is a major factor directly determining the accuracy of metrology equipments, such as optical encoders [6-8]. The SPM technique can measure the profile at a high resolution, but it is time consuming and has a critical limited range. Besides, some damages are also generated in the grating during the process of scanning measurement. Additionally, it is noted that, after the scanned signals are obtained using the SPM instruments, 
the center-of-gravity technique and Fourier transform (FT) technique are usually directly used for analysis. The FT technique cannot analyze the pitch uniformity either, though it is considered the most accurate method to measure the average period based on fundamental frequency [9]. The center-of-gravity technique is very sensitive to noise during measurement of an individual pitch. Recently, an analytical technique based on a cross-correlation filter is proposed to evaluate the grating pitches. This technique can effectively filter noise, which is very helpful in pitch evaluation [9]. In this study, we use digital image correlation (DIC) techniques to evaluate the grating pitches at a long range accurately and nondestructively. A charged-coupled device (CCD) camera is employed to acquire the grating image, and the DIC technique together with a peak detection method is used to analyze the pitch information of these images. In this measurement strategy, a CCD camera rather than SPM is used for measurement, which is time efficient and reduces the measurement cost. Besides, this technique takes full advantage of the two-dimensional (2D) information of grating images, leading to a better immunity against image noise and defects of gratings, compared with that used in Ref [9]. In the following sections, the mathematical formulations are deprived, which explain that the correlation coefficients obtained using the DIC technique retain the information of period in the original image, and the numerical simulation is conducted to show that the DIC technique can evaluate the grating pitches as accurately as the FT technique with a strong resistance to image noise. Finally, this method is applied to analyze the pitch uniformity of the grating, which is a long-range grating with a length of $145 \mathrm{~mm}$ and period of $20 \mu \mathrm{m}$, fabricated in our laboratory. 


\section{Principle}

The grating image on the CCD camera is a 2D periodical image composed of countable onedimensional (1D) integer waveforms. For simplicity, the periodical distribution is assumed as a sinusoidal function for analysis. The analytical process can be extended to other periodical distributions. Under this assumption, the grating image is shown in Fig. 1. The brightness of all the pixels along the $\mathrm{x}$-axis has the same value, while the brightness of the pixels along the y-axis conforms to a sinusoidal function. The brightness of any pixel in the image can be expressed as $F\left(x_{m}, y_{n}\right)$, i.e.,

$$
F\left(x_{m}, y_{n}\right)=A_{0}+B_{0} \sin \left(\frac{2 \pi}{P} \cdot y_{n}+\varphi_{0}\right)
$$

where, $x_{m}(1 \leq m \leq M)$ and $y_{n}(1 \leq n \leq M)$ are finite sequences of integers with $M$ and $N$ data points, respectively, corresponding to the coordinates of a pixel in the image, $P$ denotes the period of the image along the $y$-axis, $A_{0}$ is the intensity of the background light, $B_{0}$ is the amplitude of the sine function, and $\varphi_{0}$ is the initial phase, which is not important in our analysis.

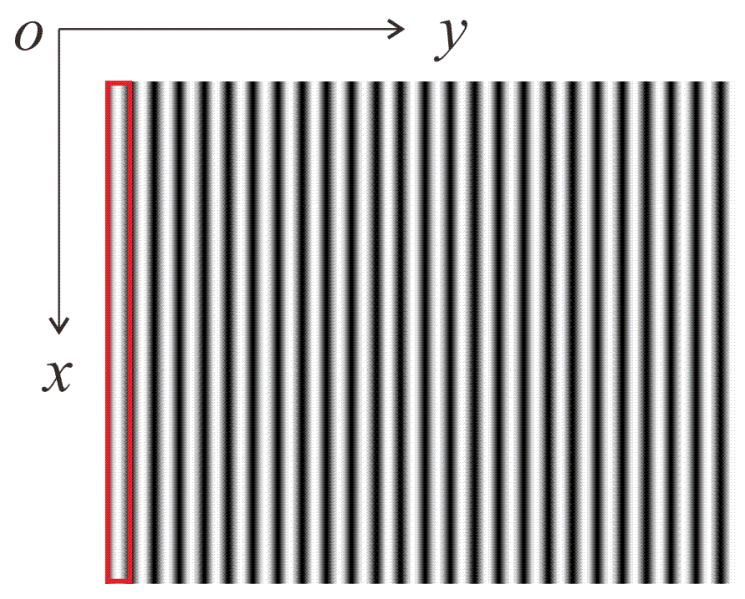

Fig. 1 Simulated grating image with the sinusoidal distribution along the y-axis.

In our simulation, the brightness of each pixel ranges from 0 to 255 . Theoretically, it is easy to evaluate the period by directly counting the number of pixels between the adjacent peaks or 
valleys. However, it is difficult to exactly determine the positions of the peaks or valleys. In order to achieve sub-pixels accuracy, the center-of-gravity technique and FT technique are usually used to analyze the period of the signal. However, the images acquired using the sensor are always accompanied with different types of noises. As a result, the center-of-gravity method causes a large error. Meanwhile, the FT technique cannot determine the uniformity of the period. In this study, we introduce the DIC technique to filter the image prior to the period evaluation. The DIC technique was first presented independently by Yamaguchi and Peters [10] to obtain the material characteristics from image deformation, but it seems that the DIC technique has never been applied for the evaluation of grating metrology before. In this study, it is used as a convenient approach to evaluate the periodic information of grating images. The algorithm is conducted in three steps. First, the image of the first period in the original image is considered as a template, known as the reference image shown in the red square in Fig. 1. It can be expressed as

$$
F_{0}=F\left(x_{m}, y_{n}\right) \quad 1 \leq x_{m} \leq M, 1 \leq y_{n} \leq P_{T}
$$

where, $P_{T}$ is the number of pixels in one period of the image at an accuracy of one pixel, which can be obtained easily using software. Second, the template is used for correlation with the following image successively, and the correlation coefficient $r(k)$ is recorded.

$$
r(k)=F_{0}\left(x_{m}, y_{n}\right) * F\left(x_{m}, y_{n}+k\right)
$$

where, ${ }^{*}$ denotes the correlation operation, and $k$ is an integer representing the displacement between two images. As the correlation coefficients show a local maximum when the two images are most similar, $r(k)$ intuitively presents the same period characters as the original image, except that most of the noise is filtered. Finally, we can investigate the period of the correlation coefficients instead of the original image to avoid interference from noises. Now, we 
prove that $r(k)$ has the same period as $F\left(x_{m}, y_{n}\right)$ from the view of mathematics. According to the definition of two-dimensional correlation, Eq. (3) can be expressed as follows,

$$
\begin{aligned}
& r(k)=\frac{\sum_{m=1}^{M} \sum_{n=1}^{P_{t}}\left[F_{0}\left(x_{m}, y_{n}\right)-\frac{1}{M \cdot P_{T}} \sum_{m=1}^{M} \sum_{n=1}^{P_{T}} F_{0}\left(x_{m}, y_{n}\right)\right] \cdot\left[F\left(x_{m}, y_{n}+k\right)-\frac{1}{M \cdot P_{T}} \sum_{m=1}^{M} \sum_{n=1}^{P_{T}} F\left(x_{m}, y_{n}\right)\right]}{\sqrt{\sum_{m=1}^{M} \sum_{n=1}^{P_{T}} F_{0}\left(x_{m}, y_{n}\right) \cdot F_{0}\left(x_{m}, y_{n}\right)-\frac{1}{M \cdot P_{T}} \sum_{m=1}^{M} \sum_{n=1}^{P_{T}} F_{0}\left(x_{m}, y_{n}\right)} \cdot \sqrt{\sum_{m=1}^{M} \sum_{n=1}^{P_{T}} F\left(x_{m}, y_{n}+k\right) \cdot F\left(x_{m}, y_{n}+k\right)-\frac{1}{M \cdot P_{T}} \sum_{m=1}^{M} \sum_{n=1}^{P_{r}} F\left(x_{m}, y_{n}\right)}} \\
& =\frac{\sum_{m=1}^{M} \sum_{n=1}^{P_{T}} F_{0}\left(x_{m}, y_{n}\right) \cdot F\left(x_{m}, y_{n}+k\right)-M \cdot P_{T} \cdot A_{0}^{2}}{\sqrt{\sum_{m=1}^{M} \sum_{n=1}^{P_{T}}\left[F_{0}\left(x_{m}, y_{n}\right) \cdot F_{0}\left(x_{m}, y_{n}\right)-A_{0}\right]} \cdot \sqrt{\sum_{m=1}^{M} \sum_{n=1}^{P_{t}}\left[F\left(x_{m}, y_{n}+k\right) \cdot F\left(x_{m}, y_{n}+k\right)-A_{0}\right]}} \\
& \square \frac{\sum_{m=1}^{M} \sum_{n=1}^{P_{T}} F_{0}\left(x_{m}, y_{n}\right) \cdot F\left(x_{m}, y_{n}+k\right)-M \cdot P_{T} \cdot A_{0}^{2}}{\sqrt{\sum_{m=1}^{M} \sum_{n=1}^{P_{T}} F_{0}\left(x_{m}, y_{n}\right) \cdot F_{0}\left(x_{m}, y_{n}\right)-M \cdot P_{T} \cdot A_{0}{ }^{2}} \cdot \sqrt{\sum_{m=1}^{M} \sum_{n=1}^{P_{T}} F\left(x_{m}, y_{n}+k\right) \cdot F\left(x_{m}, y_{n}+k\right)-M \cdot P_{T} \cdot A_{0}^{2}}}
\end{aligned}
$$

Where, the three sum terms are derived as follows,

$$
\begin{aligned}
& \sum_{m=1}^{M} \sum_{n=1}^{P_{T}} F_{0}\left(x_{m}, y_{n}\right) \cdot F_{0}\left(x_{m}, y_{n}+k\right) \\
= & \sum_{m=1}^{M} \sum_{n=1}^{P_{T}}\left[A_{0}+B_{0} \sin \left(\frac{2 \pi}{P} \cdot y_{n}+\varphi_{0}\right)\right] \cdot\left[A_{0}+B_{0} \sin \left(\frac{2 \pi}{P} \cdot\left(y_{n}+k\right)+\varphi_{0}\right)\right] \\
= & M \cdot P_{T} \cdot A_{0}{ }^{2}+\frac{1}{2} M \cdot B_{0}{ }^{2} \sum_{n=1}^{P_{T}}\left[\cos \left(\frac{2 \pi}{P} \cdot k\right)-\cos \left(\frac{2 \pi}{P} \cdot\left(2 y_{n}+k\right)+2 \varphi_{0}\right)\right] \\
= & M \cdot P_{T} \cdot A_{0}{ }^{2}+\frac{1}{2} M \cdot B_{0}{ }^{2}\left[P_{T} \cos \left(\frac{2 \pi}{P} \cdot k\right)-\sum_{n=1}^{P_{T}} \cos \left(\frac{2 \pi}{P} \cdot\left(2 y_{n}+k\right)+2 \varphi_{0}\right)\right] \\
\square & M \cdot P_{T} \cdot A_{0}{ }^{2}+\frac{1}{2} M \cdot B_{0}{ }^{2} \cdot P_{T} \cos \left(\frac{2 \pi}{P} \cdot k\right) \\
& \sum_{m=1}^{M} \sum_{n=1}^{P_{T}} F\left(x_{m}, y_{n}+k\right) \cdot F\left(x_{m}, y_{n}+k\right) \\
& =\sum_{x=1}^{M} \sum_{y=1}^{P_{T}} F_{0}\left(x_{m}, y_{n}\right) \cdot F_{0}\left(x_{m}, y_{n}\right) \\
& =\sum_{x=1}^{M} \sum_{y=1}^{P_{T}}\left[A_{0}+B_{0} \sin \left(\frac{2 \pi}{P} \cdot y_{n}+\varphi_{0}\right)\right] \cdot\left[A_{0}+B_{0} \sin \left(\frac{2 \pi}{P} \cdot y_{n}+\varphi_{0}\right)\right] \\
& =M \cdot P_{T} \cdot A_{0}{ }^{2}+M \cdot B_{0}{ }^{2} \cdot \sum_{y=1}^{P_{T}} \sin \left(\frac{2 \pi}{P} \cdot y_{n}+\varphi_{0}\right) \cdot \sin \left(\frac{2 \pi}{P} \cdot y_{n}+\varphi_{0}\right) \\
& =M \cdot P_{T} \cdot A_{0}{ }^{2}+\frac{1}{2} M \cdot B_{0}{ }^{2} \cdot \sum_{y=1}^{P_{T}}\left[1-\cos \left(\frac{2 \pi}{P} \cdot 2 y_{n}+2 \varphi_{0}\right)\right] \\
& \square M \cdot P_{T} \cdot A_{0}{ }^{2}+\frac{1}{2} M \cdot P_{T} \cdot B_{0}{ }^{2}
\end{aligned}
$$

We have assumed that $P_{T}=P$ during the derivation process for simplicity. A very simple expression is obtained when we substitute Eqs. (5) and (6) into Eq. (4), 


$$
r(k)=\cos \left(\frac{2 \pi}{P} \cdot k\right)
$$

From Eq. (7), it is noted that the correlation function $r(k)$ is a cosine sequence. It has the period $P$, which is the same as $F\left(x_{m}, y_{n}\right)$, although the amplitude changes to one and the initial phase changes to zero. In other words, the correlation function $r(k)$ consist information of the period in $F\left(x_{m}, y_{n}\right)$, which helps us analyze the periodic features of the original image. The analytical method used in Ref [9] is also easily applied to process one row signals of the grating image or the signals gathered using the one-dimension photoelectric detector instead of the CCD camera. However, the method used in Ref [9] requires a template having the same period as the signal, and only $1 \mathrm{D}$ signals are used for analysis. If the period of the template is different from that of the signals, it will affect the accuracy of results. In our case, we directly select a part of the twodimensional original image as a template, which can be used to extract the periodical information more accurately. Meanwhile, our method takes full advantage of the information in twodimensional images, which will provide results that are less influenced with noise. In addition, our method can be easily developed to analyze the pitches of the 2D grating. Another advantage in our study is that we use a CCD camera to acquire the grating image, while the method described in Ref [9] uses a metrological atomic force microscope (AFM) to probe the grating, which is time consuming to perform one measurement, and it cannot be used in the grating evaluation with a long range. Although the resolution of the CCD camera is lower than that of the AFM, it does provide us a convenient and fast method to evaluate the grating uniformity, and its accuracy is sufficient in most applications if an appropriate method is used to analyze the grating signals. Therefore, our technique is more suitable for metrological grating uniformity evaluation. 


\section{Simulation}

\subsection{Ideal Grating image}

The period of the grating fabricated in our laboratory is $20 \mu \mathrm{m}$. When it is imaged on the CCD camera by an objective lens with a magnification of $10 \times$, the period of the grating image is about 50 pixels. In order to obtain a realistic simulation, we generate an image with sinusoidal distribution along the y-axis, as shown in Fig. 1, whose period is 50 pixels. After the correlation coefficient $r(k)$ is obtained, the grating pitch can be defined as the distance between its adjacent peak-positions or valley-positions. As is shown in Fig. 2, the individual pitch is calculated using the following relation:

$$
\left\{\begin{array}{c}
d_{1}=D_{1}-D_{0} \\
d_{2}=D_{2}-D_{1} \\
\vdots \\
d_{j}=D_{j}-D_{j-1} \\
\vdots \\
d_{L}=D_{L}-D_{L-1}
\end{array}\right.
$$

The average pitch is given by:

$$
d_{a v r}=\frac{1}{L} \sum_{j=1}^{L} D_{j}
$$

The uniformity of pitches is calculated using:

$$
u=\max \left\{\operatorname{abs}\left\{d_{j}-d_{i}\right\}\right\}
$$

We can see that $u$ is determined using the maximum pitch difference, which lacks statistical significance and is sensitive to noise or surface defects of the grating. For evaluating the grating pitches with better statistical performance, another parameter i.e., standard deviation should be used. It denotes the degree of deviation from the average value. For normal distribution, the 
standard deviation $\delta$ indicated that there are $99.7 \%$ pitches of the grating located in the range from $\left(d_{a v r}-3 \delta\right)$ to $\left(d_{a v r}+3 \delta\right)$. Its definition is as follows,

$$
\delta=\sqrt{\frac{1}{L} \sum_{j=1}^{L}\left(d_{j}-d_{a v r}\right)^{2}}
$$

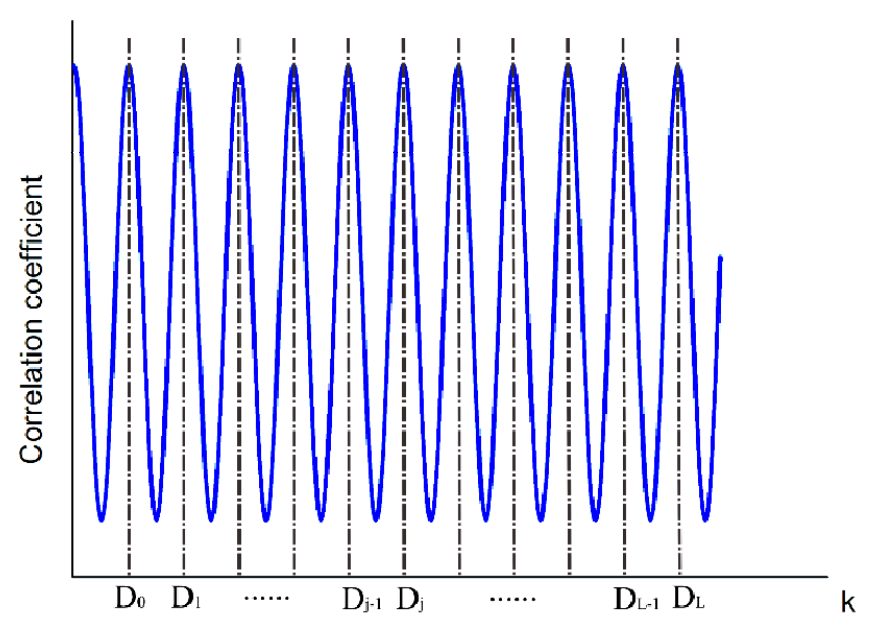

Fig. 2 Definition of pitches.

The grating image acquired using the CCD camera is always accompanied with different types of noises, which may have a great effect on the peak-position of the coefficients. For evaluating the peak-position more accurately, three methods are applied to determine the peak-position instead of selecting the position of the local maximum value as the peak-position, and their accuracy is compared with each other. One method is to select the middle position of the full width at half maximum (FWHM) in the crest as the peak-position. Another method is to select the position of the maximum value of the quadratic curve (QC) fitted from the crest using the least square method. In addition, the other method is to select the gravity center (GC) of the crest. As is shown in Fig. 3, the position of the red circle is selected as the peak-position. 

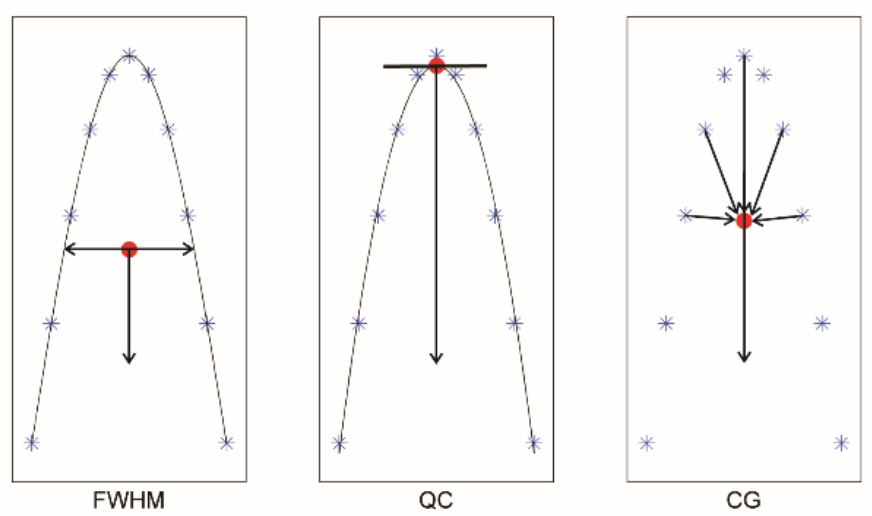

Fig. 3 The positions of red points are selected as peak-positions using three methods.

In simulation, the results calculated using the DIC technique are compared with that of the FT techniques, which is proposed by Gaoliang Dai [1]. Only the average values are shown because the FT technique cannot provide the information of pitch uniformity. The FT technique used in our simulation and experiment can be implemented using the following two steps. First, the FT technique is used to calculate the period according to each row signal in the original image. Therefore, the number of these periods calculated in one image is equal to the number of rows in the image. Second, the average value of these periods is the final period calculated using the FT technique. In an ideal circumstance, there are no noise and no aberrations in the grating image, as is shown in Fig. 1. Both the DIC and FT techniques can achieve a high accuracy. Table 1 shows the pitch average values calculated using the DIC and FT techniques. It is noticed that the results calculated using the FT technique have some deviation from the true value due to the frequency resolution, while the DIC technique can evaluate the average period without any error in this simulation.

Table 1. Average pitches and corresponding error calculated using different methods.

\begin{tabular}{ccccc}
\hline Technique & \multicolumn{5}{c}{ DIC } & FT \\
\cline { 1 - 4 } Method* & FWHM & QC & GC & 49.995995 \\
\hline davr@ & 50 & 50 & 50 & 0.004005 \\
\hline Error\& & 0 & 0 & 0 & \\
\hline
\end{tabular}


* The method of determining the peak-position

@ davr means average period, unit (pixels)

\& Error represents the deviation between average period calculated and true period, unit (pixel)

In the deduction of Eq. (7), we have assumed that $P_{T}=P$ for simplicity. Now, we will discuss

the influence on the correlation coefficients by simulation when $P_{T} \neq P$. The image is generated with a size of $1024 \times 1280$ pixels, and the average periods calculated using different methods that deviate from the true period are shown in Fig.4. The average pitches calculated using the DIC technique vary near zero, while that calculated using the FT technique change periodically. In addition, we can see that the results obtained using the FT technique change with lager range when the period increases. This is because the number of periods decreases when the period increases for an image with constant size due to the energy spectrum leakage. Therefore, pitch evaluation using the DIC technique has advantages over the FT technique in our measurement. By comparison, among all the methods used to determine the peak-position, the DIC technique together with the FWHM method seems to give the most accurate and stable average period in this case. It does not depend on the value of the true period of the image, and thus, we mainly use it to analyze the grating images in our measurement.

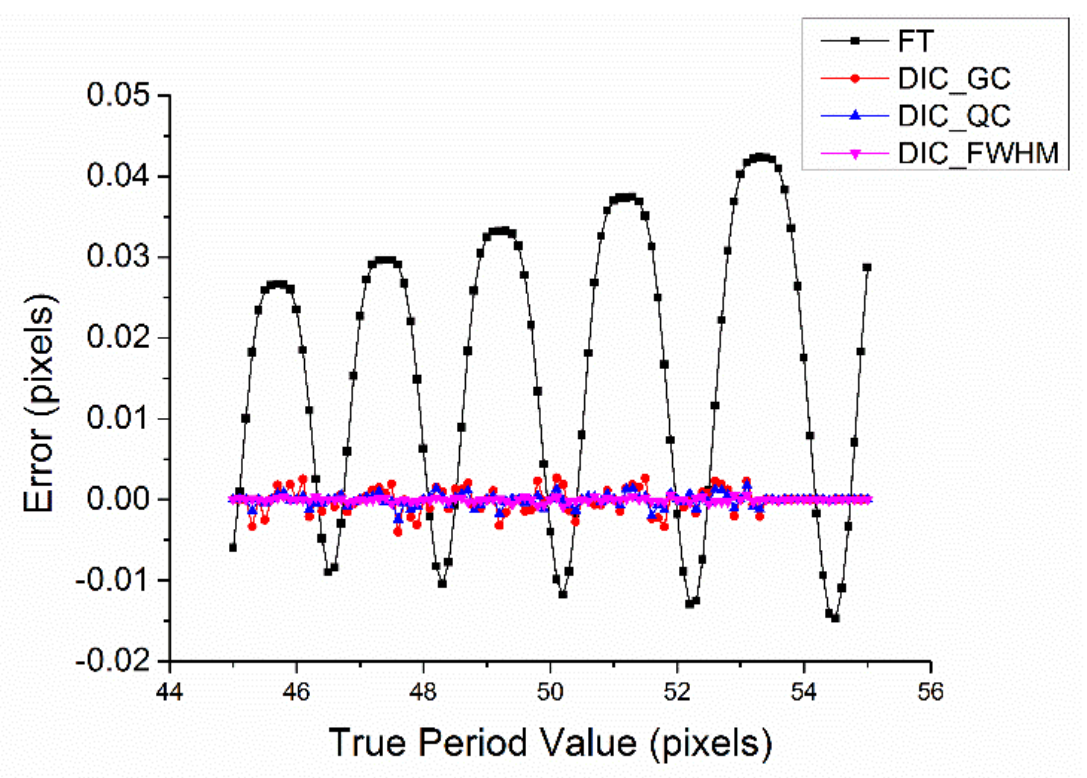


Fig. 4 Errors of pitch average calculated using different methods vary with the true value of period of the grating image. The vertical axis denotes the difference between the average period and true period calculated using different methods, and the horizontal axis denotes the true value of the period.

\subsection{Grating image with noises}

Because of the surface blemish of grating, asymmetrical distribution of light-beam, contamination of lens, and detects of wires, the grating image always contains all types of noises, which may cause the brightness to change irregularly. The distribution of brightness is deviated from the sinusoidal distribution and contains noise, although it still maintains the periodical distribution.

The noise may cause some errors to our calculating results. In order to investigate how the average pitch calculated using the DIC technique has a good agreement with the FT technique, the simulations are performed using Matlab, in which the noises with different distributions are added into the image with a period of 50 pixels and a size of $1024 \times 1280$ pixels. The image of the first period is obtained as a template for correlation with others, and the peak-positions of the correlation coefficient are detected using three methods. Eventually, the average period, $d_{a v r}$, evaluated using different methods is calculated. The simulation is repeated 200 times at each noise with a standard deviation $\delta$ and peak-to-valley uniformity $u$. Table 2 shows the results of the calculated average period from the image with Gaussian noise, speckle noise, and salt and pepper noise, and Fig. 5 shows the simulated grating images with different noises. Both the Gaussian and speckle noises have a distribution with zero mean value. However, the former has a standard deviation of 0.05 , while the later has a standard deviation of 0.5 , which is almost the highest intensity allowed in our programs. The salt and pepper noise has a density of $10 \%$. Finally, the signals combined with all the above noises and the non-uniformity of the light beam 
are added to the ideal grating image. Fig. 6 shows how the DIC technique filters noise in the image shown in Fig. 5(d). Note that the noises and non-uniformity of the light beam only have an influence on the amplitude of the correlation coefficients, whose phase is almost immune to these factors. From the data shown in Table 2, we find that the final results calculated using the DIC technique are similar to the true values as well as the FT technique. All these methods provide the average period with an accuracy of up to 0.01 pixels from the true value, which is accurate enough in our measurement.

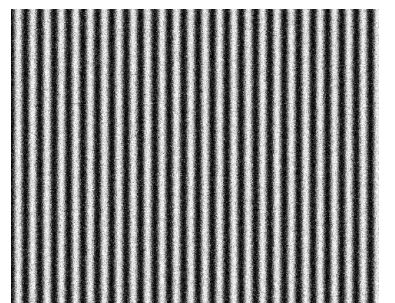

(a)

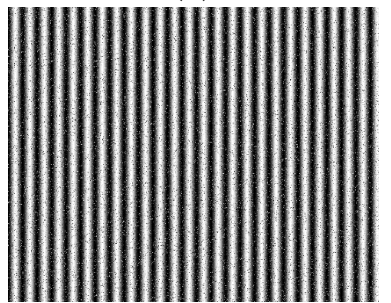

(c)

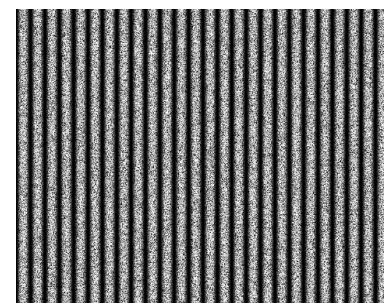

(b)

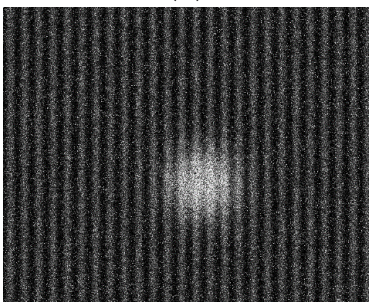

(d)

Fig. 5 Simulated grating image added with different noises. (a) Gaussian noise with zero mean and 0.05 standard deviation, (b) speckle noise with zero mean and 0.5 standard deviation, (c) salt and pepper noise with 0.1 density, and (d) a combination of Gaussian noise with 0.02 standard deviation, speckle noise with 0.2 standard deviation, salt and pepper noise with 0.02 density, and non-uniform illumination. 


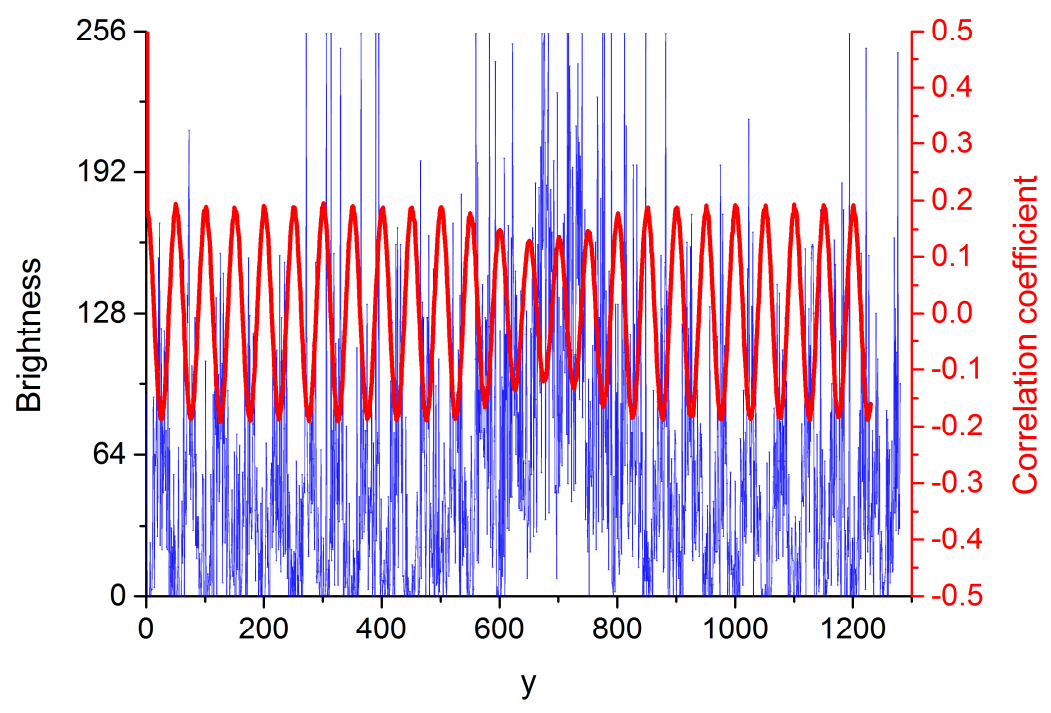

Fig. 6 The blue line denotes the brightness of the middle row pixels in grating image shown in Fig. 5. (d). The red line denotes the correlation coefficient.

So far, the mathematical theory and numerical simulation confirm that the DIC technique can not only evaluate the pitch average as accurately as the FT technique but also provide uniformity of pitches even if the amount of noise in the image is high.

Table 2 Simulation results and corresponding standard deviation and uniformity.

\begin{tabular}{|c|c|c|c|c|c|}
\hline \multirow{2}{*}{ Noise Type } & & \multicolumn{3}{|c|}{ DIC } & \multirow{2}{*}{ FT } \\
\hline & & FWHM & $\overline{\mathrm{QC}}$ & $\mathrm{GC}$ & \\
\hline \multirow{3}{*}{ Gaussian noise } & $d_{\text {avr }}$ & 49.999993 & 49.999987 & 50.000017 & 49.996384 \\
\hline & $u$ & 0.008068 & 0.006636 & 0.006659 & 0.003727 \\
\hline & $\delta$ & 0.001605 & 0.001317 & 0.001317 & 0.003675 \\
\hline \multirow{3}{*}{ speckle noise } & $d_{\text {avr }}$ & 50.000038 & 50.000005 & 50.000017 & 49.996021 \\
\hline & $u$ & 0.013781 & 0.017472 & 0.017143 & 0.005913 \\
\hline & $\delta$ & 0.002817 & 0.002633 & 0.002903 & 0.004127 \\
\hline \multirow{3}{*}{$\begin{array}{l}\text { salt and pepper } \\
\text { noise }\end{array}$} & $d_{\text {avr }}$ & 50.000096 & 50.000018 & 50.000019 & 49.996498 \\
\hline & $u$ & 0.008337 & 0.006186 & 0.006152 & 0.002531 \\
\hline & $\delta$ & 0.001455 & 0.001114 & 0.001135 & 0.003536 \\
\hline \multirow{3}{*}{$\begin{array}{l}\text { Combination } \\
\text { of different } \\
\text { noises }\end{array}$} & $d_{\text {avr }}$ & 50.00088 & 50.000312 & 50.00039 & 49.998495 \\
\hline & $u$ & 0.0452728 & 0.033325 & 0.038251 & 0.008538 \\
\hline & $\delta$ & 0.008656 & 0.00563 & 0.005994 & 0.002385 \\
\hline
\end{tabular}




\section{Experiments and Results}

The optical system is shown in Fig. 7. Light beam is collimated with a lens and illuminates the grating fabricated in our laboratory on the displacement stage, which has a travel range of 300 $\mathrm{mm}$ and a resolution of $0.25 \mu \mathrm{m}$. The grating is imaged on a CCD camera $(1024 \times 1280$ pixels and pixel pitch is $5.2 \mu \mathrm{m}$ ) through the objective lens with a numerical aperture (NA) of 0.25 . One sample of the grating images is shown in Fig. 8. The grating grooves and grating ridges in the image can be observed. The CCD camera sends the image to a personal computer for analysis as soon as it acquires the grating image. Eventually, the DIC technique is used to evaluate the grating pitches.

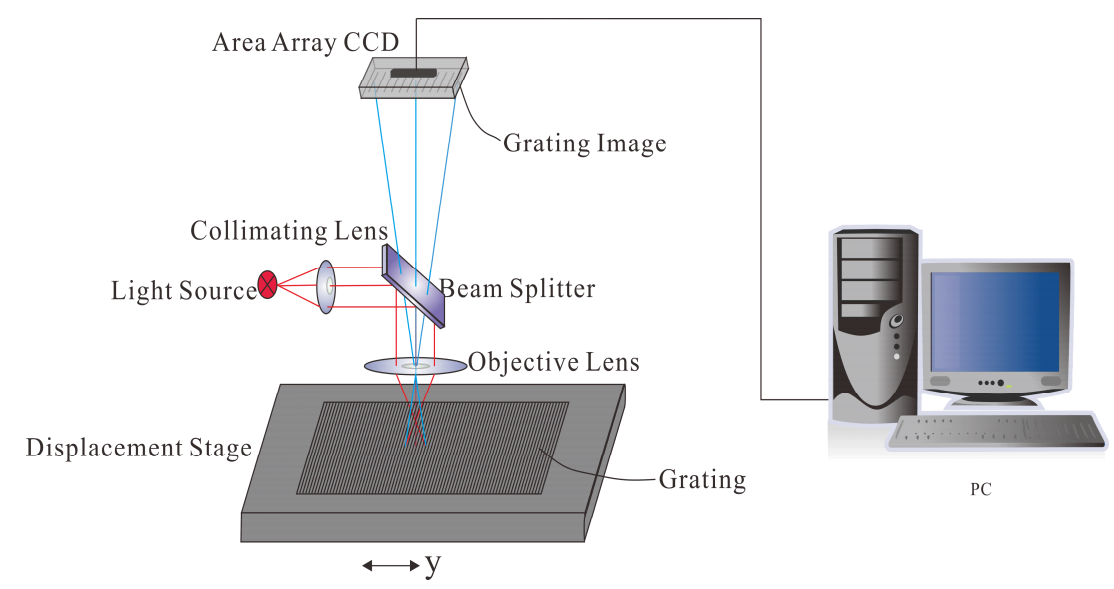


Fig. 7 Schematic of the experimental setup.

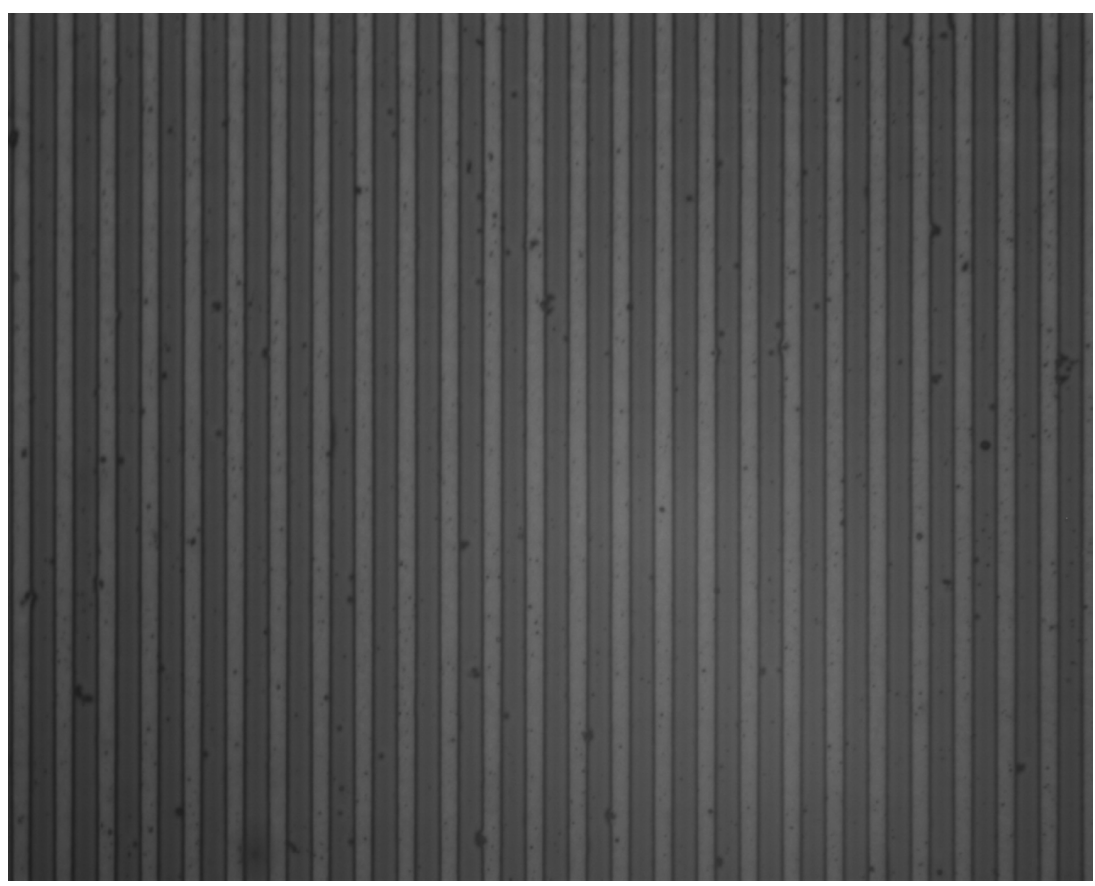

Fig. 8 One sample of the grating images in the measurement.

\subsection{The first evaluation method of grating uniformity}

While the displacement stage with a long-range grating is moving with constant speed, the CCD camera takes frames, and the DIC technique is used to calculate the pitches in the image. After all pitches in one frame are obtained, we take out a particular pitch and record it. As shown in Fig. 9, the shadow pitch is recorded in each frame. During the measurement, the speed of the displacement stage matches with the frame rate of the CCD camera. When the CCD camera takes two frames, the grating moves right by just one pitch. Therefore, continuous pitches of the grating are obtained. 


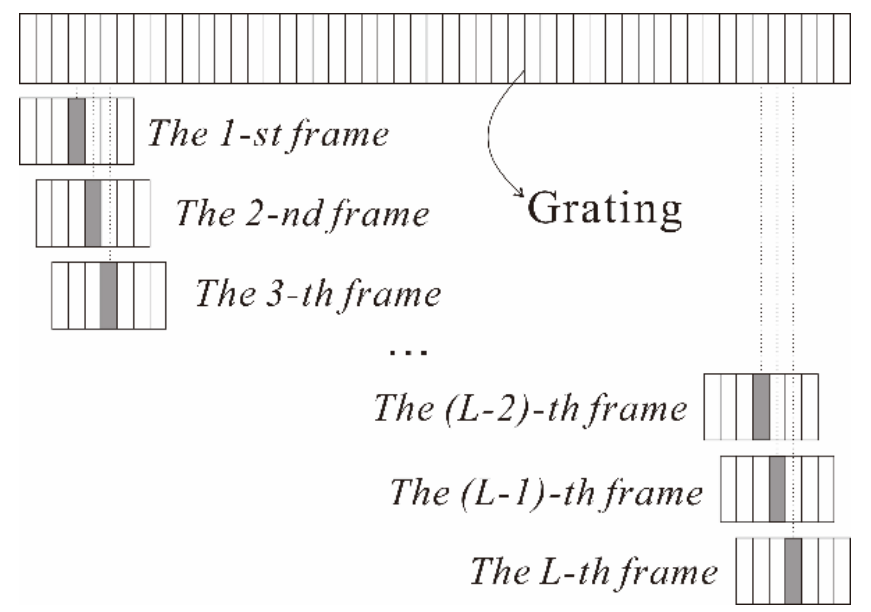

Fig. 9 The first method to acquire the individual pitch of the entire grating.

Five sets of measurements are taken during the same range of grating under the same condition. Although our grating is as long as $145 \mathrm{~mm}$, we only measure a 35 -mm-region of the grating because the images become defocus out of this region. The five sets of the measurement results are shown Fig. 10, and the detail results are listed in Table 3. For convenience, the unit of pitch has been changed from pixel to micron. All the five sets of the results show an identical variation tendency, which verify that the variation is mainly caused by the grating pitches rather than the random errors, such as image noise and environmental effects. However, in the position of $15 \mathrm{~mm}$, the grating pitches deviate almost by one pixel from the other pitches. The two reasons for this phenomenon are: 1) the grating pitches deviate from others on this position, and 2) there are some damages or large amount of dust on this local place, which has some influence on the results. By monitoring the grating image on the CCD camera, a large damage is found on the grating of the position of about $15 \mathrm{~mm}$. Hence, we assume that this is the main reason for the irregular change in the result.

All five measurements demonstrate that the pitch peak-to-valley uniformity is less than 812 $\mathrm{nm}$. The standard deviation is less than $36 \mathrm{~nm}$, which means that there are $99.7 \%$ of pitches located in the range of $(20.000 \pm 0.108) \mu \mathrm{m}$. This accuracy of $108 \mathrm{~nm}$ is the three times the 
standard deviation of the assumed-Gaussian distribution, which might be uncertain due to the limited resolution of the discrete pixels of the CCD camera.

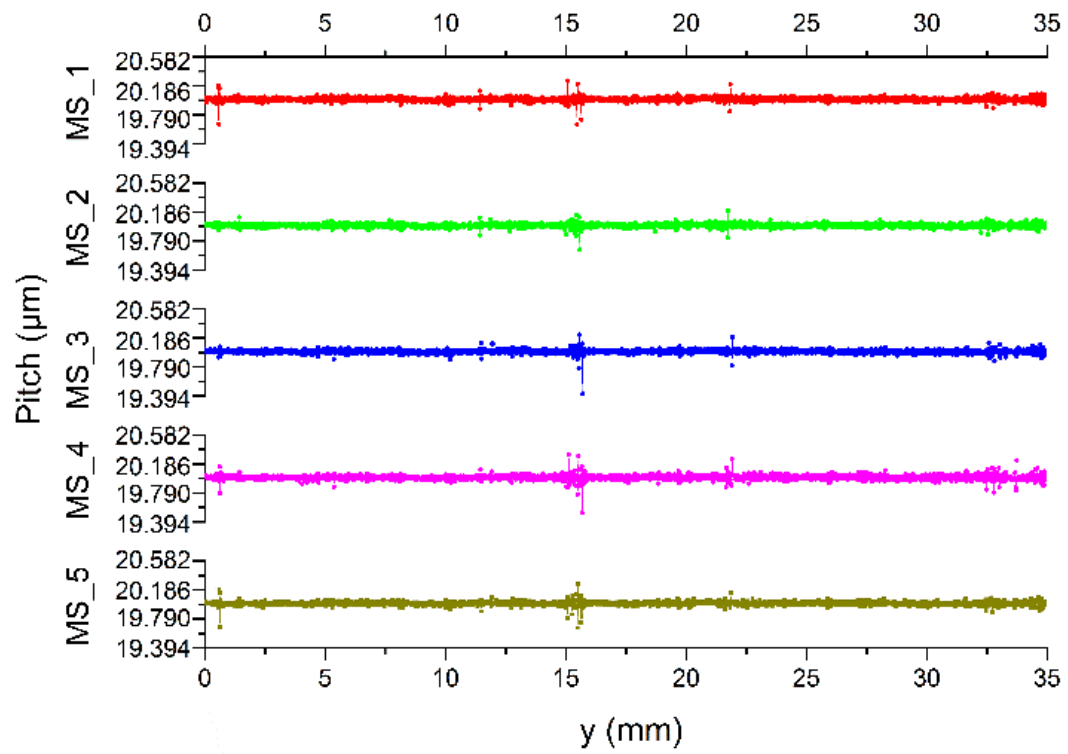

Fig. 10 Measurements results of grating pitches in the 35-mm-region using the first evaluation pattern.

Table 3. Five sets of measurement results calculated using the DIC technique with FWHM method using the first pattern (unit: $\mathrm{nm}$ )

\begin{tabular}{cccccc}
\hline & MS_1 & MS_2 & MS_3 & MS_4 & MS_5 \\
\hline $\mathrm{u}$ & 594 & 538 & 812 & 800 & 594 \\
$\delta$ & 28 & 24 & 28 & 36 & 28 \\
\hline
\end{tabular}

\subsection{The second evaluation method of grating uniformity}

The second method also uses the process shown in Fig. 9 to acquire the grating images. The only difference from the first method is that, we record the average pitch of the pitches in one frame and consider it as the pitch on the corresponding position of the grating. The reasons for this are listed below. First, the method fabricating gratings is to use the multiple repeat of the parallel Dammann writing facility $[11,12]$. Thus, it seems that the grating has a very high uniformity in local place. Meanwhile, in the field of microscope, only a small scope, about $408 \times 510 \mu \mathrm{m}$ area, can be observed. Approximately 25.5 strips appear in one grating image on the CCD camera. 
Thus, we suppose that the grating pitches in one image share the same value. Second, the pixels on the CCD camera are discrete. As we know, even if the grating is strictly uniform, its pitches of the image on the CCD camera also show a slightly difference between one and another due to the discrete pixels, aberration, and noises. Therefore, for higher precision, we select the average value of one frame of the grating image as the period in this local place. In this way, we can decrease this type of errors to the least level. So the more accurate measurement results will be obtained by using this method than the first method.

The five measurement results are shown in Fig. 11, and the detail results of these measurements are listed in Table 4, which shows that the grating has a peak-to-valley uniformity of $48 \mathrm{~nm}$ with standard deviation of $4 \mathrm{~nm}$, and there are $99.7 \%$ of pitches located in the range of $(20.000 \pm 0.012) \mu \mathrm{m}$. This accuracy of $12 \mathrm{~nm}$ is the three times standard deviation of the assumed Gaussian distribution, which is obtained from the averaged period over the entire grating images. This accuracy of $12 \mathrm{~nm}$ is higher than the previous accuracy of $108 \mathrm{~nm}$ due to the averaged period over the entire grating images. Again, all these five set of results show an identical variation tendency, which demonstrates the accuracy of our measurement. Another phenomenon should be noticed is that several points deviate from the main curve. If one grating pitch deviates far from others, the curve should show a slowly varying peak or valley around this position. Hence, a single point deviates from the main curve is an abnormal phenomenon. We assume that the main reason for this is that there are some damages or a large amount of dust on these grating positions. 


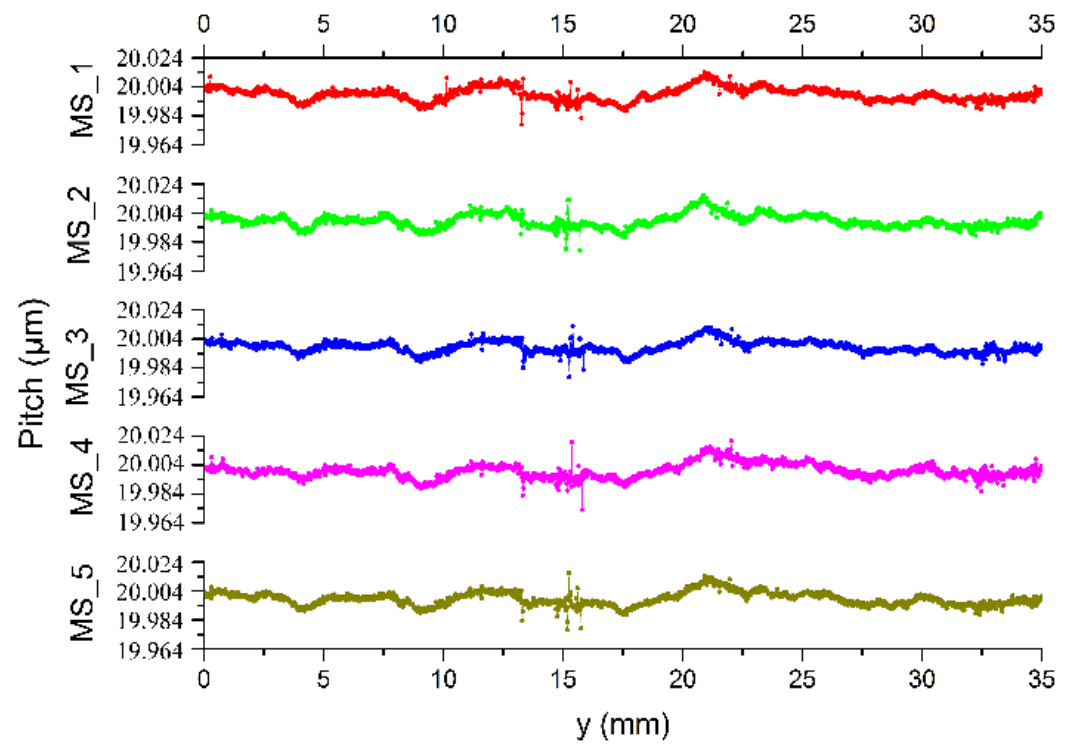

Fig. 11 Five sets of measurement results of grating pitches using the second evaluation pattern.

Table 4. Five sets of measurement results calculated using DIC technique with FWHM method by the second pattern (unit: $\mathrm{nm}$ )

\begin{tabular}{cccccc}
\hline & MS_1 & MS_2 & MS_3 & MS_4 & MS_5 \\
\hline $\mathrm{u}$ & 36 & 36 & 36 & 48 & 40 \\
$\delta$ & 4 & 4 & 4 & 4 & 4 \\
\hline
\end{tabular}

Additionally, as described above, the average period can also be calculated using the FT method, and we also can use the FT method to evaluate our grating uniformity in this method. The measurement results are shown in Fig. 12. We observe that the results show a large difference between two measurements. Therefore, we assume that the FT method is invalid in our measurement. The reasons include many aspects. First, the grating pitches do not match the size of the grating image, which causes seriously energy spectrum leakage. Second, the brightness does not strictly show sinusoidal distribution or rectangular distribution, and the background light is not constant in the image, which leads to a serious error in the FT technique when calculating the average period. Third, our grating may have a slightly different duty cycle in different positions of the grating due to the unstable light source used to fabricate the gratings, although the grating period is still constant. This slightly different duty cycle also contributes to 
the error in the FT technique. In other words, the FT technique is not suitable to be applied in this case. Most of the change in the curves shown in Fig. 12 is caused by the FT technique. However, in the position of $10 \mathrm{~mm}, 13 \mathrm{~mm}$, and $15 \mathrm{~mm}$, the curve show identical peaks, which are mainly caused by the difference in the duty cycle.

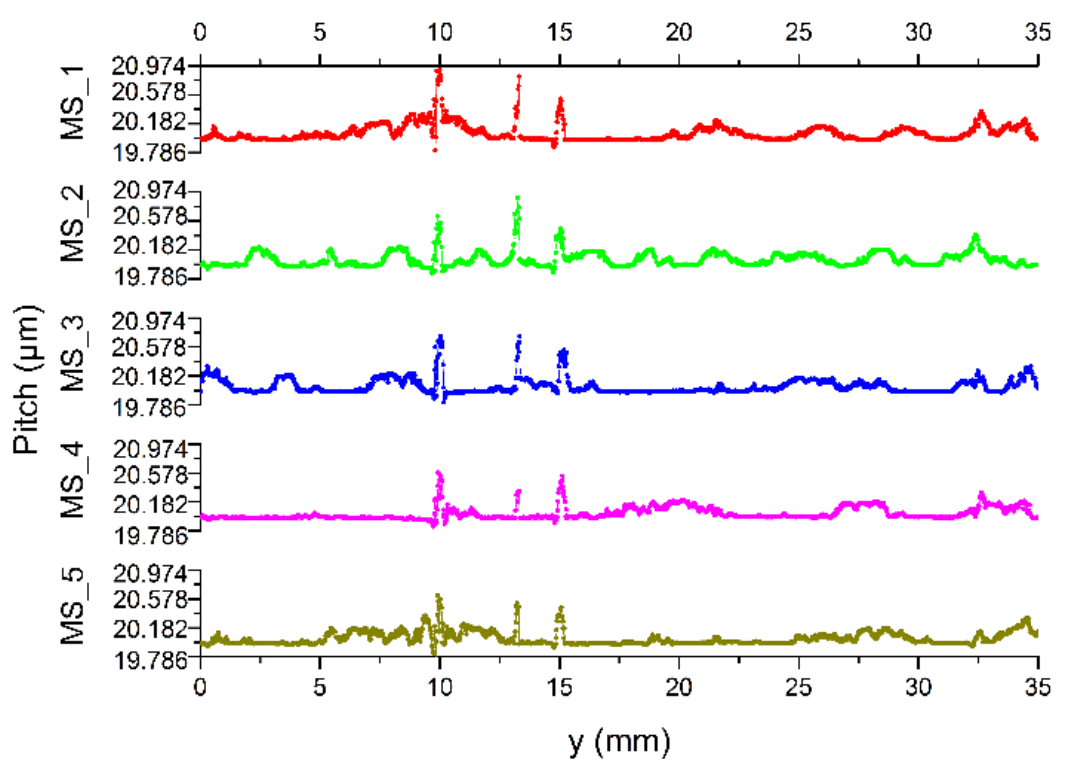

Fig. 12 Five sets of measurement results of grating pitches using FT method.

\subsection{The third evaluation method of grating uniformity}

In the above two methods, defocus is found in the measurement, especially when the grating length is larger than $35 \mathrm{~mm}$. In order to avoid the influence of defocus, we select 30 points from the entire grating and make the grating in the focus plane at every point. Next, we take frames at the point and calculate the grating pitch. Again, five sets of measurements are performed. The results are shown in Fig. 13. The pitches before $90 \mathrm{~mm}$ are a little larger than that after $90 \mathrm{~mm}$. It can be seen that the peak-to-valley uniformity is about $32 \mathrm{~nm}$, which shows that our grating has a good uniformity on the order of dozens of nanometers at a range of $145 \mathrm{~mm}$. 


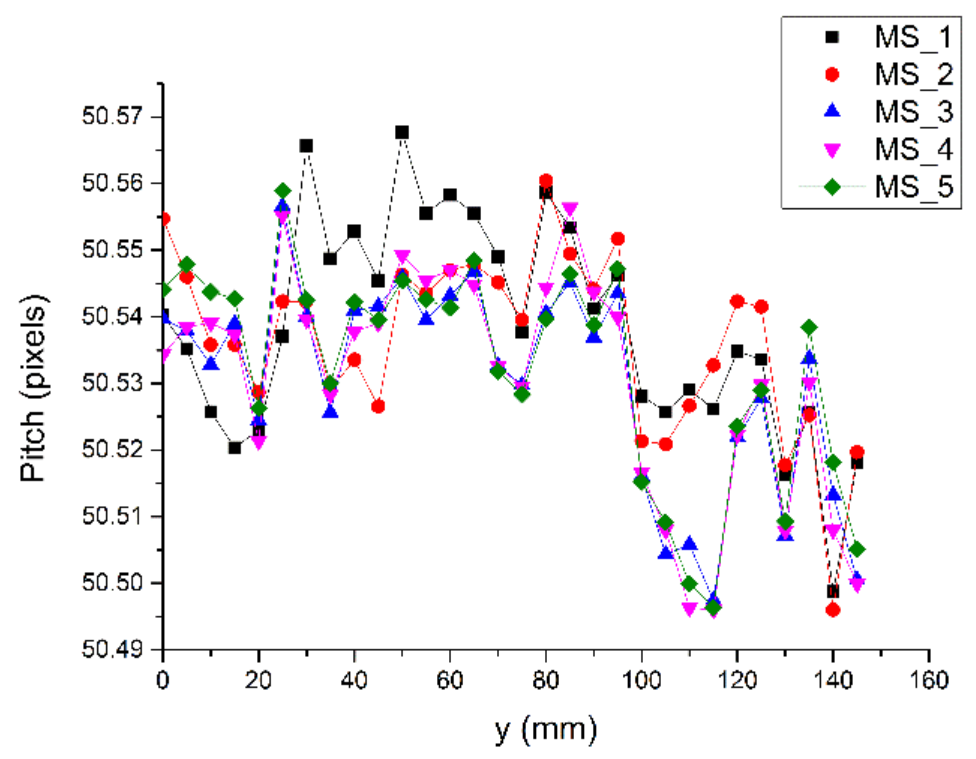

Fig. 13 Average pitches at a long range.

To the best of our knowledge, a metrology analysis of grating with excellent uniformity is seldom reported, partly because it is difficult to scan the entire grating pitches at a long range using the usual scanning probe instruments, and it is hard to exactly isolate and identify every sources from the mixed systematic and random errors. In our measurement scheme, the systematic errors include at least the following factors. First, the grating vector is not strictly parallel to the direction of movement, which renders the measurement results different from their real values. Second, the discrete pixels of the CCD camera limit the resolution of the grating images, resulting in limited accuracy of the pitch measurements. Third, the aberration of the image lens also makes the grating images different from the real grating. Fourth, the defect of the grating might have effects on the measurements. Fifth, non-uniformity distribution of light-beam might illuminate the grating image with different average brightness on different regions. The random errors in our measurement can be classified into low frequency noises and high frequency noises. Low frequency noises include the thermal drift, stability of light source, etc. High frequency noises are generated from electronic noise, CCD photo detector, etc. Although 
our measurements are performed on the air-floating platform in a temperature-controlled laboratory, these errors still inevitably affect the measurements. However, the DIC technique, which takes good full advantage of two-dimensional image information, helps to decrease the influence of noises and obtain the accuracy of measurement on the order of dozens of nanometers. The five sets of measurements are repeated for each evaluation method, and the corresponding results have an identical variation tendency, demonstrating the measurement data is credible. The first evaluation method might have low accuracy due to the discrete pixels of the CCD camera and other systematic errors. The third evaluation method cannot describe the entire pitches of the grating though it does not suffer from defocus. The second evaluation method is more reasonable to evaluate the entire grating pitches at a high accuracy, which demonstrates that the grating has a peak-to-valley uniformity of $48 \mathrm{~nm}$ with a standard deviation of $4 \mathrm{~nm}$ during the range of $35 \mathrm{~mm}$. Thus, the DIC technique provides a cheap, simple, time efficient, and accurate method to evaluate the grating pitches, which is highly essential for improvement of grating fabrication.

\section{Conclusion}

The digital image correlation technique with the peak-position detection method is used to evaluate the pitches of one-dimensional grating. A CCD camera is used to acquire the grating images. The mathematical formulation and simulation with different noises certify that this technique can evaluate the uniformity of grating pitches with a high accuracy. The results demonstrate that our grating has a peak-to-valley uniformity of $48 \mathrm{~nm}$ with a standard deviation of $12 \mathrm{~nm}$. This work demonstrated to be useful for evaluation of the pitch uniformity for metrological gratings. 


\section{Funding Information}

National Science Foundation (NSF) (61127013, 61405214, 61308073)

\section{References}

1. G. Dai, L. Koenders, F. Pohlenz, T. Dziomba and H.-U. Danzebrink, Accurate and traceable calibration of one-dimensional gratings, Measurement Science and Technology. 16 (2005) 12411249.

2. F. Meli, "WGDM-7: Preliminary comparison on Nanometrology: Nano4: 1D gratings," Final report, OFMET Wabern. 30 (2000).

3. S. S. H. Naqvi, S. Gaspar, K. Hickman, K. Bishop and J. R. Mcneil, Linewidth Measurement of Gratings on Photomasks - a Simple Technique, Appl. Opt. 31 (1992) 1377-1384.

4. F. Meli and R. Thalmann, Long-range AFM profiler used for accurate pitch measurements, Measurement Science and Technology. 9 (1998) 1087-1092.

5. H. Kunzmann, T. Pfeifer, and J. Flugge, Scale Vs Laser Interferometers Perfomance and comparison of two meauring systems, Annals of the CIRP. 42 (1993) 753-767.

6. . Y. Lee, H. L. Hsieh, G. Lerondel, R. Deturche, M. P. Lu and J. C. Chen, Heterodyne grating interferometer based on a quasi-common-optical-path configuration for a two-degrees-of-freedom straightness measurement, Appl. Opt. 50 (2011) 1272-1279.

7. F. Cheng and K. C. Fan, Linear diffraction grating interferometer with high alignment tolerance and high accuracy, Appl. Opt. 50(2011) 4550-4556.

8. J. Guan, P. Köchert, C. Weichert and R. Tutsch, A high performance one-dimensional homodyne encoder and the proof of principle of a novel two-dimensional homodyne encoder, Prec. Eng. 37 (2013) 865-870.

9. X. Chen and L. Koenders, A novel pitch evaluation of one-dimensional gratings based on a crosscorrelation filter, Measurement Science and Technology. 25(2014) 044007. 
10. I. Yamaguchi, Speckle displacement and decorrelation in the diffraction and image fields for small object deformation, Journal of Modern Optics. 28(1981) 1359-1376.

11. Feng Zhu, Jianyong Ma, Wei Huang, Jin Wang, and Changhe Zhou, Parallel laser writing system with scanning Dammann lithography, Chin. Opt. Lett. 12(2014) 080501-080504.

12. Wei Huang, Jianyong Ma, Feng Zhu, Jin Wang, and Changhe Zhou, Low divergent diffractive optical element for remote detection, Chin. Opt. Lett. 12 (2014) 070501. 